\title{
Einleitung zum Themaheft Raum und Landwirtschaft
}

In der schweizerischen Landwirtschaft haben in den letzten drei Jahrzehnten bedeutende betriebswirtschaftliche Umstellungen eine teilweise Anpassung an die sich ändernden (wirtschaftlichen) Verhältnisse sowie beachtliche Produktivitätssteigerungen bewirkt: Mechanisierung und Intensivierung führten zu einer Hebung der Arbeits- bzw. Flächenproduktivität. Der Trend zur betrieblichen Vereinfachung und Spezialisierung des Produktionsprofils - z. T. auf alternative Produktionszweige - brachte den landwirtschaftlichen Betrieben eine teilweise Angleichung an die naturräumlichen Bedingungen sowie an die Situation auf den Beschaffungs- und Absatzmärkten.

Trotz dieser intensiven Anpassungsbemühungen vermochte die Landwirtschaft nicht in gleichem Maß wie die übrigen Wirtschaftssektoren am volkswirtschaftlichen Wachstum teilzuhaben. Sie gerät damit in ein Spannungsfeld, das v.a. durch intersektorale und intrasektorale (Einkommens-)Disparitäten sowie einen Bedeutungs- und Funktionswandel gekennzeichnet ist.

Von zentraler Bedeutung in der agrarpolitischen Diskussion ist der Abbau der intrasektoralen Disparitäten. Die regionalen Struktur-, Produktivitäts- und Einkommensunterschiede zeigen, daß die Veränderungen innerhalb der Landwirtschaft hinsichtlich Intensität und zeitlichem Ablauf unterschiedlich verliefen. Strukturstarke Regionen des Mittellandes und strukturschwache Räume im Berggebiet sind hier etwa als Extremfälle zu nennen.

Im Zusammenhang mit der erwähnten Problematik kommt demnach dem räumlichen Standort der landwirtschaftlichen Unternehmung eine grundlegende Bedeutung zu. Dabei können sowohl ein einzelner Landwirtschaftsbetrieb oder aber auch mehrere (z. B. alle) Betriebe einer Region als Standort aufgefaßt werden. Jeder Standort weist eine spezifische Kombination verschiedener natur- und wirtschaftsräumlicher Standortfaktoren auf, die bezüglich Qualität, Verfügbarkeit und ev. Preis räumlich variieren. Für sämtliche Betriebe einer Region sind dies:

- Naturräumliche Ausstattung der Region

- Situation auf den (regionalen) Beschaffungsmärkten für Produktionsfaktoren sowie andere Güter und Dienstleistungen

- Persönlichkeitsmerkmale der Betriebsleiter der Region

- Situation auf den (regionalen) Absatzmärkten (v.a. räumliche Lage, Produzentenpreise)

- Politisch-institutionelle Bedingungen für die Betriebe der Region

- Soziale Umwelt und volkswirtschaftlicher Entwicklungsstand
Die Standortansprüche der einzelnen Produktionszweige und die bereits erwähnten lokalen Standortfaktoren - insbesondere die daraus realisierbaren Kosten, Preise und Erträge - bestimmen im wesentlichen Art und Menge der agrarischen Produktion. Grundsätzlich vermögen lediglich jene Betriebe ein ausreichendes Einkommen zu erzielen, deren Produktionsprofil auf die naturräumliche Ausstattung des Standortes und die Verhältnisse auf den Beschaffungs- und Absatzmärkten abgestimmt ist.

Im Laufe der Zeit wurden eine ganze Reihe verschiedener Ansätze zur Erfassung räumlich differenzierter Strukturen und Prozesse entwickelt (vgl. Beitrag RUPPERT).

Grundlage vieler inneragrarischer Spannungen bilden die Problemdisparitäten zwischen einzelnen Regionen (z. B. Mittelland/Berggebiet). Der Abbau des Polarisierungstrends, welcher einerseits $\mathrm{zu}$ intensiv bewirtschafteten Produktionsräumen und andrerseits $\mathrm{zu}$ agrarischen Problemgebieten führt, ist Gegenstand agrarpolitischer Diskussionen (vgl. Beitrag RIEDER). Eine zentrale Rolle bei den vielfältigen Entscheidungsprozessen innerhalb der landwirtschaftlichen Unternehmung spielt der Betriebsleiter. Unterschiedliche Standortbedingungen führen möglicherweise $\mathrm{zu}$ räumlich differenzierten Verhaltensmustern hinsichtlich Standortanpassung, Investitionsfreudigkeit usw. (vgl. Beitrag GANTNER). Zur Sicherung der vielfältigen landwirtschaftlichen Funktionen bedarf es der gemeinsamen Bemühungen von Agrar- und Regionalpolitik. Die langfristige Bereitstellung ausreichender Produktionsflächen in standortgünstiger Lage bildet die unabdingbare Voraussetzung für eine effiziente Produktion (vgl. Beitrag SCHMID).

Wie aus dem vorliegenden Heft zu ersehen ist, kann das aktuelle Problem der intrasektoralen Disparitäten aus verschiedenen Blickwinkeln analysiert, gewertet und der agrarpolitischen Diskussion zugeführt werden. Neben Agronomen, Ökologen und Kulturingenieuren vermögen auch Geographen in Form von theoretischen Ansatzpunkten, empirischen Befunden sowie regionalpolitischen Anregungen einen Beitrag zu leisten.

Zum Schluß möchte ich allen Autoren für ihre bereitwillige Mitarbeit bei der Realisierung dieses Themaheftes der GEOGRAPHICA HELVETICA herzlich danken.

Regula Volkart-Fürrer, Dr.

Geographisches Institut Universität Zürich-Irchel Winterthurerstr. 190, 8057 Zürich 